\title{
Ampullary Gland
}

National Cancer Institute

\section{Source}

National Cancer Institute. Ampullary Gland. NCI Thesaurus. Code C77955.

Paired exocrine glands of the male reproductive system located at the medial termination of the ductus deferens that produce and secrete lipids and glycogen, components of the seminal fluid. 